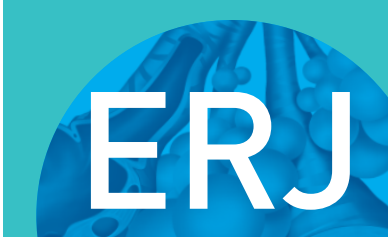

open research
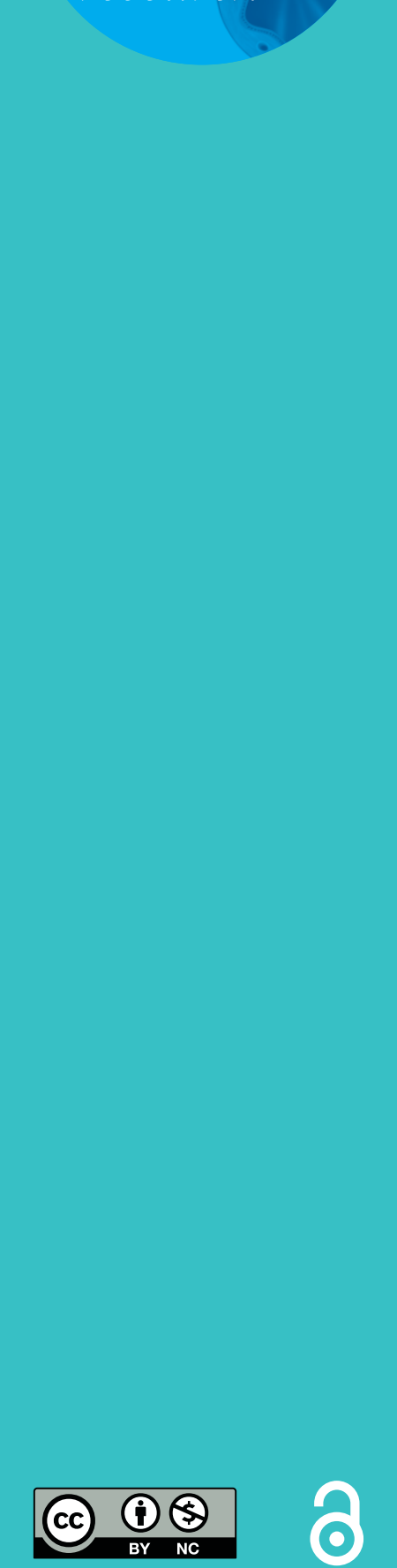

\section{Pulmonary vascular pruning in smokers with bronchiectasis}

\author{
Alejandro A. Diaz ${ }^{1}$, Diego J. Maselli ${ }^{2}$, Farbod Rahaghi ${ }^{1}$, Carolyn E. Come ${ }^{1}$, \\ Andrew Yen ${ }^{3}$, Erick S. Maclean ${ }^{1}$, Yuka Okajima ${ }^{1}$, Carlos H. Martinez ${ }^{4}$, \\ Tsuneo Yamashiro ${ }^{5}$, David A. Lynch ${ }^{6}$, Wei Wang ${ }^{7}$, Gregory L. Kinney (1) ${ }^{8}$, \\ George R. Washko ${ }^{1}$ and Raúl San José Estépar ${ }^{9}$
}

Affiliations: ${ }^{1}$ Division of Pulmonary and Critical Care Medicine, Brigham and Women's Hospital, Harvard Medical School, Boston, MA, USA. ${ }^{2}$ Division of Pulmonary Diseases and Critical Care, University of Texas Health Science Center, San Antonio, TX, USA. ${ }^{3}$ Dept of Radiology, University of California, San Diego, CA, USA. ${ }^{4}$ Division of Pulmonary and Critical Care Medicine, University of Michigan Health System, Ann Arbor, MI, USA. ${ }^{5}$ Dept of Radiology, Graduate School of Medical Science, University of the Ryukyus, Nishihara, Japan. ${ }^{6}$ Dept of Radiology, National Jewish Health, Denver, CO, USA. ${ }^{7}$ Division of Sleep Medicine, Brigham and Women's Hospital, Harvard Medical School, Boston, MA, USA. ${ }^{8}$ Colorado School of Public Health, University of Colorado Denver, Aurora, CO, USA. 'Dept of Radiology, Brigham and Women's Hospital, Harvard Medical School, Boston, MA, USA.

Correspondence: Alejandro A. Diaz, Division of Pulmonary and Critical Care Medicine, Dept of Medicine, Brigham and Women's Hospital, 75 Francis Street, Boston, MA 02115, USA.

E-mail: ADiaz6Rabwh.harvard.edu

ABSTRACT There are few studies looking at the pulmonary circulation in subjects with bronchiectasis. We aimed to evaluate the intraparenchymal pulmonary vascular structure, using noncontrast chest computed tomography (CT), and its clinical implications in smokers with radiographic bronchiectasis.

Visual bronchiectasis scoring and quantitative assessment of the intraparenchymal pulmonary vasculature were performed on CT scans from 486 smokers. Clinical, lung function and 6-min walk test (6MWT) data were also collected. The ratio of blood vessel volume in vessels $<5 \mathrm{~mm}^{2}$ in cross-section (BV5) to total blood vessel volume (TBV) was used as measure of vascular pruning, with lower values indicating more pruning. Whole-lung and lobar BV5/TBV values were determined, and regression analyses were used to assess the differences in BV5/TBV between subjects with and without bronchiectasis.

155 (31.9\%) smokers had bronchiectasis, which was, on average, mild in severity. Compared to subjects without bronchiectasis, those with lower-lobe bronchiectasis had greater vascular pruning in adjusted models. Among subjects with bronchiectasis, those with vascular pruning had lower forced expiratory volume in $1 \mathrm{~s}$ and 6MWT distance compared to those without vascular pruning.

Smokers with mild radiographic bronchiectasis appear to have pruning of the distal pulmonary vasculature and this pruning is associated with measures of disease severity.

@ERSpublications

Smokers with radiographic bronchiectasis have intraparenchymal pulmonary vascular pruning, which is associated with both lung function and exercise capacity impairment http://ow.ly/YCYm30mgV7z

Cite this article as: Diaz AA, Maselli DJ, Rahaghi F, et al. Pulmonary vascular pruning in smokers with bronchiectasis. ERJ Open Res 2018; 4: 00044-2018 [https://doi.org/10.1183/23120541.000442018].

This article has supplementary material available from openres.ersjournals.com

Received: March 172018 | Accepted after revision: Oct 072018

Copyright $\odot$ ERS 2018. This article is open access and distributed under the terms of the Creative Commons Attribution Non-Commercial Licence 4.0. 


\section{Introduction}

Bronchiectasis is a pathological dilation of the airways characterised by repeated cycles of infection and inflammation [1]. This airway abnormality is associated with a myriad of conditions including chronic obstructive pulmonary disease (COPD) [2], and linked to increased healthcare utilisation and mortality [3].

Subjects with other chronic airway diseases such as COPD have changes in the intraparenchymal pulmonary vessels including remodelling and pruning $[4,5]$. While these changes in the pulmonary circulation might be extrapolated to smokers with bronchiectasis as they share common risk factors for bronchial and vascular injury such as smoking and inflammation, there is limited information on the intraparenchymal pulmonary circulation in adults with bronchiectasis. In an early study, RYLAND and REID [6] demonstrated that children with cystic fibrosis, a disease characterised by the development of bronchiectasis and right ventricle hypertrophy, have fewer pulmonary arteries per unit area of lung tissue. Furthermore, a reduced calibre of the intraparenchymal arteries was documented in infants with cystic fibrosis compared to controls [7], a finding we have also shown in adult smokers with bronchiectasis (versus nonbronchiectasis subjects) on computed tomography (CT) scans [8]. These studies, however, did not provide data on the entire in vivo pulmonary vascular tree, an evaluation that is now possible to perform noninvasively using CT techniques [4,9]. Using these techniques, the pulmonary vascular tree can be analysed and regional differences examined. The assessment of the intraparenchymal vasculature might enhance the understanding of bronchiectasis in smokers and inform potential interventions.

In this study, we used data from the COPDGene study [10] to quantitatively assess the intraparenchymal pulmonary vessels in smokers with radiographic bronchiectasis and test the hypothesis that this airway abnormality is associated with clinically relevant vascular pruning.

\section{Methods}

\section{Study population}

We used baseline data from the COPDGene study, which was designed to determine genetic and epidemiological determinants of COPD [10]. Briefly, current and former smokers ( $\geqslant 10$ pack-years) aged 45-80 years were recruited to this study. Subjects with an active pulmonary disease other than COPD and asthma, and those who had an acute respiratory disease episode (i.e. new or increase in respiratory symptoms) within a month prior to enrolment were excluded. To test our hypothesis, we used the data from the first 500 COPDGene smokers in whom bronchiectasis scoring and pulmonary vascular data collection were performed. All subjects provided written informed consent to participate in the study. The institutional review board at each participating clinical centre approved the COPDGene study and the Partners HealthCare Research Committee (2007P-000554) approved the current study.

\section{CT reading}

COPDGene imaging protocols were provided elsewhere [10]. Pertinent to this study is that the reconstruction of the volumetric images had submillimetre slice thickness, allowing a detail assessment of the bronchi. The readings were performed on baseline inspiratory CT scans by two pulmonologists with experience in lung imaging and two chest radiologists using the following steps. First, each CT was read by two primary readers independently. Bronchiectasis on CT was coded as yes, no or equivocal. A second reading was conducted on all CT scans by three primary readers. Then, all CT scans in which there were disagreements on the presence of bronchiectasis between the primary readers were assigned for a third reading to a single chest radiologist who assigned the final code. Finally, after these steps were concluded, all CTs with bronchiectasis were scored by the primary readers. Inter-reader reproducibility assessment of bronchiectasis presence and the total score was performed on $32 \mathrm{CT}$ scans by the three primary readers.

\section{Bronchiectasis definition and scoring}

The presence of bronchiectasis was defined with one or more of the following criteria: 1) airway dilation (airway lumen diameter greater than adjacent pulmonary vessel diameter), 2) abnormal airway tapering of any extent (no decrease in or increase in lumen moving from proximal to distal airways) and 3) visualisation of a bronchus within $1 \mathrm{~cm}$ of the pleura. As the definition of bronchiectasis in terms of the number of locations varies, our primary definition of bronchiectasis case was defined with at least one bronchial path (i.e. one bronchopulmonary segment) involved. Alternate definitions used for the main analysis were as follows: 1) two or more bronchopulmonary segments involved, and 2) both criteria 2 and 3 above present. The severity of bronchiectasis was assessed using a previously used score sheet [8]. Briefly, the severity scoring is based on the degree of airway dilation, the type of bronchiectasis, the number of airway paths affected, and the presence of wall thickness and mucous plugging in the bronchiectatic airways. The score ranges from 1 to 40 with a greater number indicating greater severity. We use the Netter's bronchial path (bronchopulmonary segment) nomenclature, which has 10 and eight 
paths (segments) for the right and left lung, respectively [11]. The assessment at bronchial path level allowed us to then score bronchiectasis for the whole lung and each lobe.

\section{Vascular morphology}

The methodology to identify and quantify the pulmonary vessels was described elsewhere $[4,9]$. In brief, a scale-space particles method was used to segment and extract the vascular tree. The total intraparenchymal blood vessel volume (TBV) of the combined arteries and veins was determined. The distal pulmonary vasculature was determined as the aggregate volume of vessels whose cross-sectional area was $<5 \mathrm{~mm}^{2}$ (BV5). We used the ratio of distal to total blood vessel volume as a measure of vascular pruning (BV5/ $\mathrm{TBV} \times 100$ ) [4]. We report this metric for the whole lung and lung lobes (right upper lobe, right middle lobe, right lower lobe, left upper lobe and left lower lobe). This latter approach allowed us to compare the BV5/TBV between bronchiectatic and nonbronchiectatic lobes.

\section{Lung volume and emphysema}

Total lung volume at full inspiration was used as a radiological measure of total lung capacity (TLCCT) and was expressed as a percentage of predicted values [12]. Emphysema was measured as the percentage of low-attenuation areas below -950 Hounsfield units (\%LAA-950) [13]. \%LAA-950 is reported for the whole lung and upper and lower lobes of combined lungs. Subjects were defined as having emphysematous or nonemphysematous lungs when \%LAA-950 of the lower third was $\geqslant 5 \%$ or $<5 \%$, respectively [14].

\section{Clinical and physiological assessments}

Demographic and clinical data were collected with standardised questionnaires, which are available at www.COPDGene.org [10]. A subject was considered to have chronic heart failure and coronary artery disease if they responded "yes" to the question, "Have you ever been told by a physician that you have [condition name]?". Positive responses about heart attack, angina and coronary artery disease as well as

\section{TABLE 1 Demographic and clinical characteristics of the subjects by bronchiectasis status}

\section{Subjects without bronchiectasis}

Subjects
Age years
Females
Non-Hispanic white race
Height cm
Body mass index kg-m ${ }^{-2}$
Current smoking status
Smoking history pack-years
Chronic heart failure
Coronary artery disease
FEV1 L
FEV $1 \%$ predicted
FVC L
FVC \% predicted
TLCcT L
TLCCT \% predicted
6-min walk test distance m
Resting SaO $\%$
Spirometric COPD
Whole-lung TBV mL
Whole-lung BV5 mL
Whole-lung BV5/TBV \%
Whole-lung \%LAA-950 \%
Upper-lobe \%LAA-950 \%
Lower-lobe \%LAA-950 \%

331
$60 \pm 9$
$57 \%$
$78 \%$
$168 \pm 9$
$29 \pm 6$
$41 \%$
$45 \pm 25$
$1.8 \%$
$10 \%$
$2.2 \pm 0.8$
$77 \pm 24$
$3.3 \pm 0.9$
$88 \pm 17$
$5.5 \pm 1.4$
$97 \pm 17$
$428 \pm 119$
$96 \pm 3$
$47 \%$
$277 \pm 61$
$167 \pm 41$
$60 \pm 6$
$6.9 \pm 9.8$
$7.8 \pm 10.8$
$5.5 \pm 8.0$

Data are presented as mean \pm SD, unless otherwise stated. Missing data: total lung capacity determined by computed tomography (TLCCT), $n=4$; TLCCT $\%$ predicted, $n=4 ; 6$-min walk test, $n=2$; whole-lung percentage of low-attenuation areas below -950 Hounsfield units (\%LAA-950), $n=4 ; \%$ LAA-950 of the upper and lower lobes, $n=4$. $F E V 1$ : forced expiratory volume in $1 \mathrm{~s} ; \mathrm{FVC}$ : forced vital capacity; $\mathrm{SaO}_{2}$ : arterial oxygen saturation; COPD: chronic obstructive pulmonary disease; TBV: total blood vessel volume; BV5: blood vessel volume in vessels $<5 \mathrm{~mm}^{2}$ in cross-section. 
self-reported history of angioplasty or coronary artery bypass surgery were collapsed into one condition named coronary artery disease. Spirometric measures of lung function were performed before and after the administration of albuterol per the American Thoracic Society recommendations. Post-bronchodilator forced expiratory volume in $1 \mathrm{~s}(\mathrm{FEV})$ and forced vital capacity are presented as percentages of the predicted values [15]. Exercise capacity was measured using the 6-min walk test (6MWT) and prior to starting the test, resting oxygen saturation $\left(\mathrm{SaO}_{2}\right)$ was also determined [16].

\section{Statistical analysis}

Measurements are presented as mean $\pm \mathrm{SD}$ or $\mathrm{n}(\%)$. Comparisons between smokers with and without bronchiectasis as well as those with high versus low CT bronchiectasis score were performed using t-test, nonparametric test or Chi-squared test as appropriate. The differences in BV5/TBV between lung lobes with and without bronchiectasis were further adjusted for potential confounders using multivariable regression analysis. Covariates for these models were chosen a priori, and included age, sex, race, pack-years smoked, FEV1 \% predicted, TLCCT \% predicted, $\mathrm{SaO}_{2}$, \%LAA-950 on CT and coronary artery disease. To assess the association of vascular pruning with lung function and exercise capacity, four groups were created based on the presence/absence of bronchiectasis in any lobe and intraparenchymal vascular pruning (defined as the below/above median value of the aggregate BV5/TBV from both lower lobes), and their FEV1 and 6MWT were compared using ANCOVA as those variables did not violate normality and variances across unequal samples did not show major differences. Covariates for the FEV1 model were chosen a priori, and included age, sex, race, height, pack-years of smoking, COPD status and \%LAA-950 of the lower lobes. A similar model was fitted for 6MWT, except that height was replaced by body mass index to account for body size. The intra- and inter-reader reproducibility for bronchiectasis diagnosis and severity score was assessed using kappa test and concordance correlation coefficients (CCCs), and Bland-Altman analysis [17, 18]. A p-value $<0.05$ was considered significant. SAS 9.4 software (SAS Institute, Cary, NC, USA) was used to perform the analysis.

\section{Results}

Out of 500 subjects selected, two had an equivocal diagnosis of bronchiectasis and 12 had poor-quality vascular segmentation, leaving a sample size of 486 smokers with at least one whole-lung BV5/TBV measurement. Bronchiectasis was identified in 155 (31.9\%) smokers. Compared with nonbronchiectasis subjects, those with bronchiectasis were more likely to be older and female. These subjects also had lower absolute expiratory airflows, vital capacity and 6MWT distance, with no significant differences in smoking history, cardiovascular comorbidities, resting $\mathrm{SaO}_{2}$, emphysema on CT scans, frequency of COPD and whole-lung BV5/TBV (table 1). The primary readers 1 and 3 agreed on $88 \%$ of the CTs, yielding a kappa value of 0.72 . The primary readers 1 and 2 as well as 2 and 3 agreed on $75 \%$ of the CTs, yielding a kappa value of 0.5 for both pairs. Among the cases in which the readers had an agreement, the CCC for the severity score was 0.77 (95\% CI $0.55-0.99), 0.57$ (95\% CI $0.27-0.87$ ) and 0.73 (95\% CI 0.50-0.96) for readers 2 and 3, readers 1 and 3, and readers 2 and 3, respectively. Bland-Altman analysis did not show a

\begin{tabular}{|c|c|}
\hline \multicolumn{2}{|l|}{ Bronchiectasis type } \\
\hline Cylindric & 146 (94\%) \\
\hline Varicose & $8(5) \%$ \\
\hline Cystic & $1(1 \%)$ \\
\hline \multicolumn{2}{|l|}{ Bronchiectasis location $^{\#}$} \\
\hline Right upper lobe & $19(12 \%)$ \\
\hline Right middle lobe & $97(63 \%)$ \\
\hline Right lower lobe & $81(52 \%)$ \\
\hline Left upper lobe & $53(34 \%)$ \\
\hline Left lower lobe & $62(40 \%)$ \\
\hline \multicolumn{2}{|l|}{ Number of lobes with bronchiectasis" } \\
\hline 1 & $62(40 \%)$ \\
\hline 2 & $38(25 \%)$ \\
\hline 3 & $26(17 \%)$ \\
\hline$\geqslant 4$ & $29(19 \%)$ \\
\hline CT bronchiectasis score median (IQR) & $3(2.0-5.0)$ \\
\hline
\end{tabular}


TABLE 3 Ratio of blood vessel volume in vessels $<5 \mathrm{~mm}^{2}$ in cross-section (BV5) to total blood vessel volume (TBV) by lung lobe and bronchiectasis status

\begin{tabular}{lccc} 
Lung lobe & Subjects without bronchiectasis & Subjects with bronchiectasis & p-value \\
\hline Right upper lobe & $64 \pm 7$ & $65 \pm 8$ & 0.90 \\
Right middle lobe & $54 \pm 9$ & $54 \pm 10$ & 0.91 \\
Right lower lobe & $58 \pm 7$ & $55 \pm 9$ & 0.004 \\
Left upper lobe & $62 \pm 7$ & $61 \pm 7$ & 0.87 \\
Left lower lobe & $55 \pm 9$ & $51 \pm 10$ & 0.0008
\end{tabular}

Data are presented as mean $\pm \mathrm{SD}$, unless otherwise stated. Results are from univariate comparisons of BV5/ $T B V \times 100$ between subjects with bronchiectasis in a specific lobe only and those without bronchiectasis in any lobe. Missing values: one subject had no lobar vascular measurements and one subject had no vascular measurement in the right middle lobe.

systematic bias across the bronchiectasis score range among the three primary reader pairs. The results for diagnosis of bronchiectasis by reading are shown in table E1.

A description of the CT findings is shown in table 2. Most of the subjects (94\%) had cylindrical bronchiectasis and $60 \%$ had two or more lobes affected, with the right middle lobe, right lower lobe and left lower lobe being the most commonly affected. The CT severity score was significantly higher (worse) when the disease was in the lower lobes compared to upper lobes and right middle lobe combined (5.1 \pm 4.1 versus $2.2 \pm 1.6, \mathrm{p}<0.0001$ ). Radiographic features compatible with Mycobacterium avium complex infection (Lady Windermere syndrome) was identified in eight (5.2\%) subjects with bronchiectasis.

\section{Vascular pruning in bronchiectasis subjects}

The differences in vascular pruning were apparent in the right and left lower lobes (table 3 and figure 1) but not in other lobes. Thus, BV5/TBV from both lower lobes were averaged and used for subsequent analysis. In adjusted models, the differences in BV5/TBV of the lower lobes remained significant (table 4). Analyses in the subgroups of subjects with $(\mathrm{n}=156,32.4 \%)$ and without emphysema $(\mathrm{n}=326,67.6 \%)$ showed consistent results (table 4). When the disease was defined as 1) bronchiectasis in two or more bronchopulmonary segments $(\mathrm{n}=94)$ and 2) lack of airway lumen tapering plus an airway seen within $1 \mathrm{~cm}$ of the pleura $(\mathrm{n}=61)$, the differences in BV5/TBV of the lower lobes were consistent for both the entire cohort and emphysema groups in multivariable models (table 5) regardless of the definition used.

\section{Associations of regional vascular pruning with lung function and exercise capacity}

Subjects who fell into the bronchiectasis ${ }^{+} /$vascular pruning $^{+}$group had significantly lower FEV1 $(1.70 \pm 0.70$ versus $2.24 \pm 0.81 \mathrm{~L}, \mathrm{p}<0.0001)$ and $6 \mathrm{MWT}$ distance $(361 \pm 122 \mathrm{~m}$ versus $441 \pm 98 \mathrm{~m}, \mathrm{p}<0.0001)$ than those falling in the bronchiectasis ${ }^{+} /$vascular pruning ${ }^{-}$group (figure 2, group 1 versus group 3). In adjusted models, the differences in FEV1 and 6MWT between those two groups remained significant $(\mathrm{p}<0.0001$ and $p=0.0002$, respectively). Among subjects without bronchiectasis, the differences in FEV1 and 6MWT between those with and without vascular pruning were also significant in univariate (figure 2, group 0 versus group 2) and multivariable comparisons (for all comparisons $\mathrm{p}<0.0001$ ).

\section{Discussion}

In this study, using noncontrast $\mathrm{CT}$ assessment of the intraparenchymal pulmonary vasculature in smokers with and without bronchiectasis, we demonstrated that 1) subjects with lower-lobe bronchiectasis have greater vascular pruning in those lobes than their nonbronchiectasis counterparts, and 2) subjects with bronchiectasis and pulmonary vascular pruning have more severe airflow obstruction and worse exercise capacity than those without pruning.

While airway abnormalities, such as dilation and thickening, as well as tortuosity and dilation of the bronchial arteries are well known pathological features of bronchiectasis, less is known about the intraparenchymal pulmonary circulation. Early studies in children with cystic fibrosis demonstrated the presence of smaller pulmonary artery calibre in cases versus controls and a loss of small pulmonary arteries [6,7]. In a report of 17 adult patients who underwent surgery for bronchiectasis, a lack of perfusion in bronchiectatic lung zones was documented by pulmonary angiography and histological examination showed that "the pulmonary capillary bed was not seen" [19]. In adult smokers, we previously demonstrated smaller pulmonary artery diameters in vessels associated with bronchiectatic versus nonbronchiectatic airways [8]. While these findings point to a loss of intraparenchymal pulmonary 

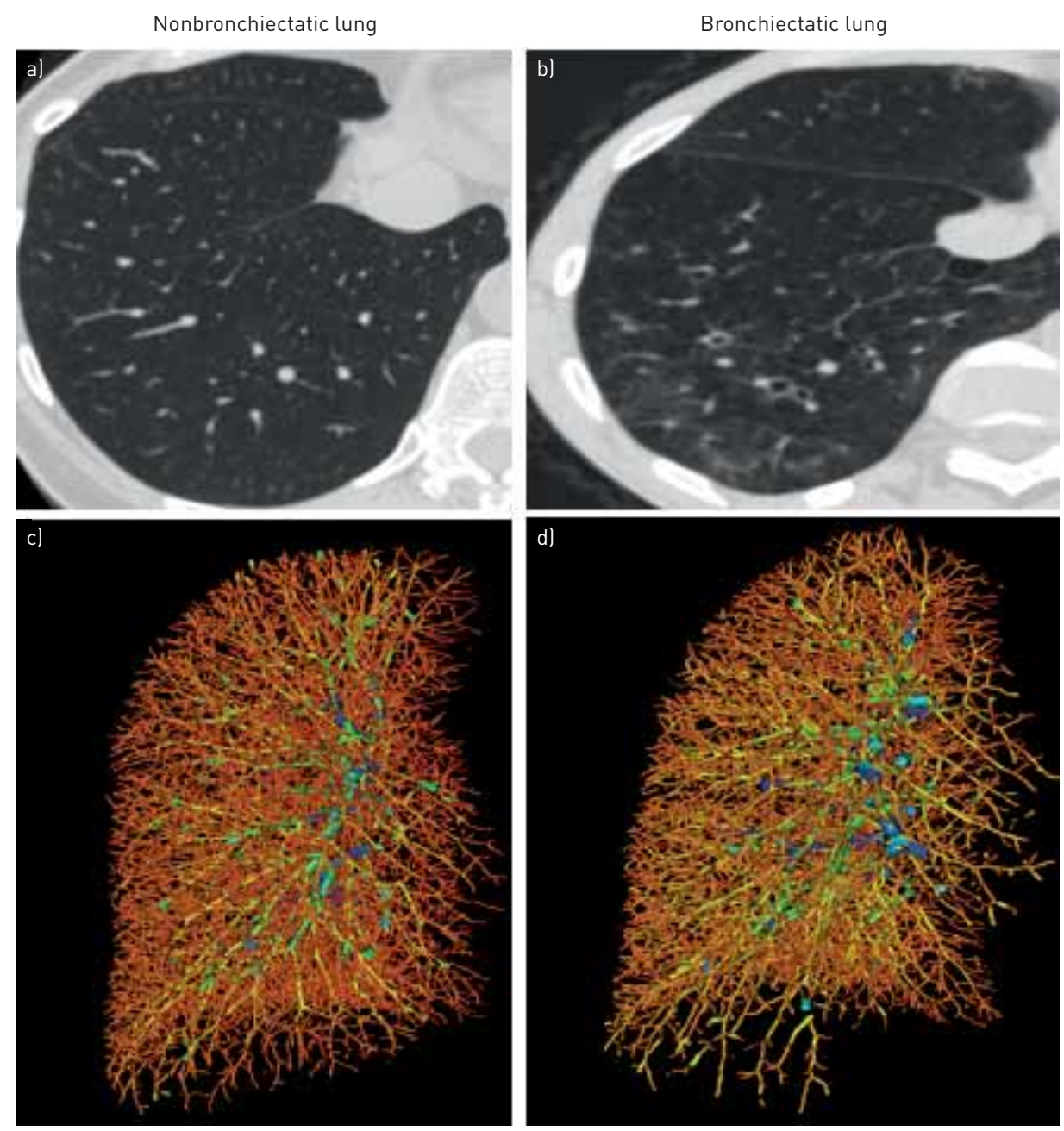

e)

$$
\text { e) }
$$

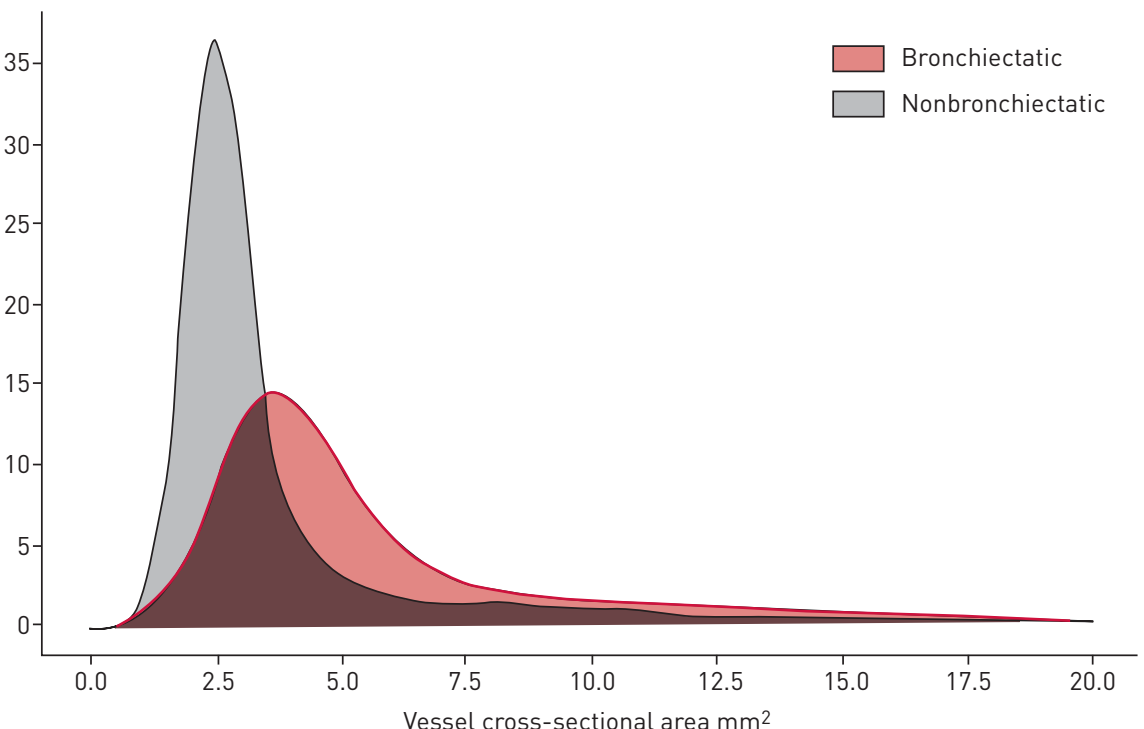

FIGURE 1 Illustration of intraparenchymal pulmonary vascular pruning in bronchiectasis. Axial computed tomography images of the right lower lobe (RLL) from subjects al without and b) with bronchiectasis. $c$ and d) Three-dimensional reconstructions of the intraparenchymal pulmonary vascular tree of the right lung from the same subjects. Note the lack of pulmonary vessels in the RLL from the subject with bronchiectasis (d). e) Plot of RLL blood vessel volume to the vessel size for both subjects. Note a lower peak in blood vessel volume of small, distal pulmonary vessels in the RLL of bronchiectatic lung compared with nonbronchiectatic lung. 
TABLE 4 Multivariable analysis for the differences in the ratio of blood vessel volume in vessels $<5 \mathrm{~mm}^{2}$ in cross-section (BV5) to total blood vessel volume (TBV) between subjects with lower-lobe bronchiectasis and without bronchiectasis

\begin{tabular}{lccc} 
Cohort/subcohort & Difference in lower-lobe BV5/TBV & SE & p-value \\
\hline Entire cohort & -1.5 & 0.35 & $<0.0001$ \\
Emphysema status subcohort & & & \\
$\quad$ Subjects with emphysema & -1.8 & 0.52 & 0.0008 \\
Subjects without emphysema & -1.6 & 0.46 & 0.0005
\end{tabular}

The differences in lower-lobe $\mathrm{BV} 5 / \mathrm{TBV} \times 100$ are from a multivariable regression model where the presence of lower lobes bronchiectasis is the main predictor. Analyses were adjusted for age, sex, race, pack-years smoked, forced expiratory volume in $1 \mathrm{~s} \%$ predicted, total lung capacity determined by computed tomography \% predicted, resting arterial oxygen saturation, percentage of low-attenuation areas below -950 Hounsfield units (\%LAA-950) of the lower lobes and coronary artery disease. For the analysis stratified by emphysema of the lower lobes, \%LAA-950 was omitted as a covariate.

vessels in bronchiectasis, further study has likely been limited but the fact that the assessments used in clinical settings are invasive or require contrast (CT angiogram). In this study, we have built on prior findings and used a noninvasive CT technique to quantify the intraparenchymal pulmonary vasculature and found that, in smokers with radiographic bronchiectasis in the lower lobes, there appears to be a loss of distal vessels. The finding suggests there might be a bronchovascular process present in mild disease as our cohort, on average, had mild radiographic bronchiectasis. Although it is not possible to identify what causes vascular pruning on CT, multiple factors, including vasoconstriction, vessel obliteration or even vessel degradation may contribute. Many of the smokers in our cohort had emphysema, a pathological feature associated with pulmonary vascular pruning. To account for emphysematous parenchymal destruction in our analyses, we used emphysema as a covariate and additionally conducted an emphysema-stratified analysis. Furthermore, we analysed vascular pruning using two additional definitions of the disease. We found that the differences in vascular pruning persisted in all those analyses, making our findings robust. We acknowledge, however, that the interplay between vascular pruning, bronchiectasis, emphysema and smoking is complex, and further research is needed to disentangle the relative contributions of these factors to vessel loss. We believe that a greater extent of this airway abnormality in addition to higher blood flow (carrying a greater burden of inflammatory mediators) in the lower lobes

TABLE 5 Multivariable analysis for the differences in the ratio of blood vessel volume in vessels $<5 \mathrm{~mm}^{2}$ in cross-section (BV5) to total blood vessel volume (TBV) between subjects with lower lobes bronchiectasis and without bronchiectasis using alternate definitions of the disease

\begin{tabular}{|c|c|c|c|}
\hline Alternate definition & Difference in lower-lobe BV5/TBV & SE & p-value \\
\hline \multicolumn{4}{|c|}{ 1) Two or more bronchial paths involved } \\
\hline Entire cohort & -1.8 & 0.40 & $<0.0001$ \\
\hline \multicolumn{4}{|l|}{ Emphysema status subcohort } \\
\hline Subjects with emphysema & -1.8 & 0.57 & 0.002 \\
\hline Subjects without emphysema & -1.3 & 0.49 & 0.009 \\
\hline \multicolumn{4}{|c|}{$\begin{array}{l}\text { 2) Lack of airway lumen tapering+airway seen } \\
\text { within } 1 \mathrm{~cm} \text { of the pleura }\end{array}$} \\
\hline Entire cohort & -2.1 & 0.58 & 0.0003 \\
\hline \multicolumn{4}{|l|}{ Emphysema status subcohort } \\
\hline Subjects with emphysema & -1.9 & 0.77 & 0.01 \\
\hline Subjects without emphysema & -2.4 & 0.80 & 0.003 \\
\hline \multicolumn{4}{|c|}{$\begin{array}{l}\text { The differences in lower-lobe } \mathrm{BV} 5 / \mathrm{TBV} \times 100 \text { are from a multivariable regression model where the } \\
\text { presence of bronchiectasis in the lower lobes is the main predictor. Analyses were adjusted for age, sex, } \\
\text { race, pack-years smoked, forced expiratory volume in } 1 \mathrm{~s} \% \text { predicted, total lung capacity determined by } \\
\text { computed tomography \% predicted, resting arterial oxygen saturation, percentage of low-attenuation areas } \\
\text { below }-950 \text { Hounsfield units (\%LAA-950) of the lower lobes and coronary artery disease. For the analysis } \\
\text { stratified by emphysema of the lower lobes, \%LAA-950 was omitted as a covariate. }\end{array}$} \\
\hline
\end{tabular}



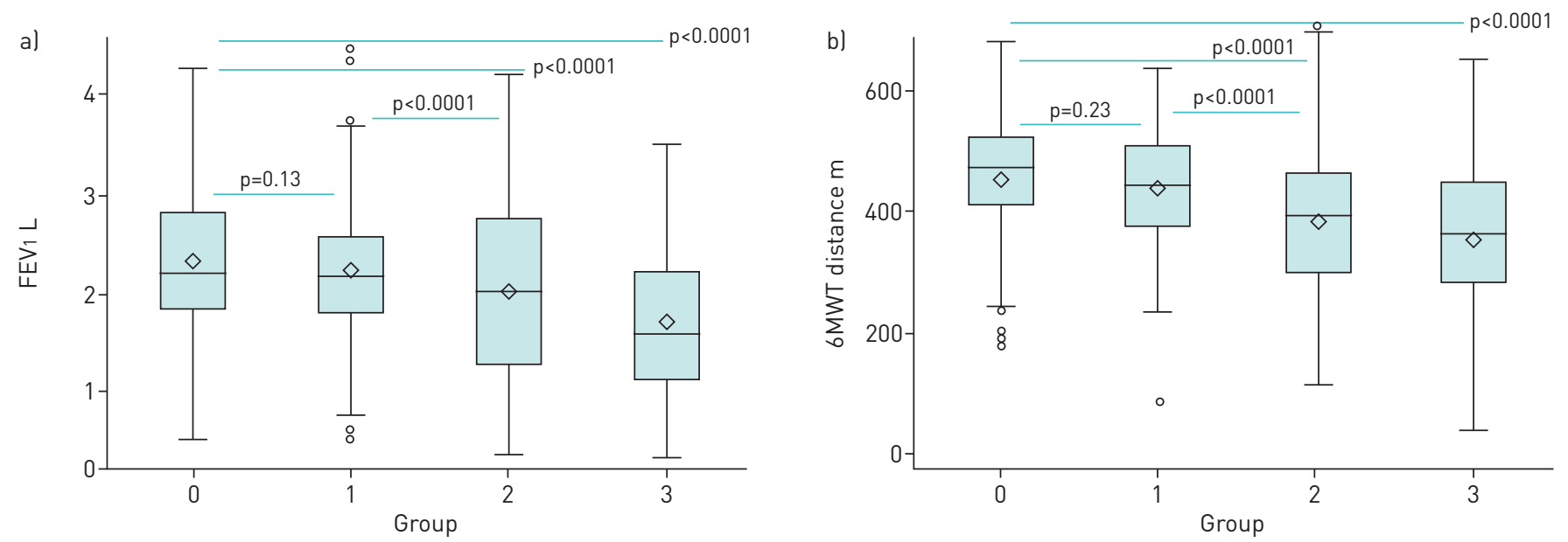

FIGURE 2 a) Forced expiratory volume in $1 \mathrm{~s}\left(\mathrm{FEV}_{1}\right)$ and b) 6-min walk test (6MWT) distance by bronchiectasis in any lobe/vascular pruning in the lower lobes groups (n=485). Group 0: bronchiectasis ${ }^{-} /$vascular pruning ${ }^{-}(\mathrm{n}=187)$; group 1: bronchiectasis ${ }^{+} /$vascular pruning $^{-}$(n=82); group 2: bronchiectasis $^{-} /$vascular pruning ${ }^{+}(\mathrm{n}=143)$; group 3: bronchiectasis $^{+} /$vascular pruning ${ }^{+}(\mathrm{n}=73$ ). Note that group 3 had lower FEV 1 and $6 \mathrm{MWT}$ distance than group 1. p-values are from univariate models. See the main text for the results of the adjusted models. One subject was excluded from these analyses because of a missing right lower lobe ratio of blood vessel volume in vessels $<5 \mathrm{~mm}^{2}$ in cross-section to total blood vessel volume, leaving a sample size of 485 . The 6MWT distance measurement was missing in two additional subjects.

may partially explain the lower-lobe predominant vascular pruning observed in our cohort. This possibility is supported by our finding showing that the disease was more severe when located in the lower lobes and an observation from a prior study demonstrating that all bronchiectatic zones with a lack of perfusion were those with the more severe form of the disease [19].

Although our data do not provide mechanistic insight into the loss of distal vessels in bronchiectasis, we believe there are several possibilities. Subjects with stable bronchiectasis have elevated systemic inflammation $[20,21]$, which might cause remodelling and distortion of the vessels potentially leading to their disappearance. An additional contributor to vascular injury in this cohort is cigarette smoking exposure. A prior CT study showed a reduced cross-sectional area of pulmonary vessels in smokers without airflow obstruction [22]. Finally, the intraparenchymal vascular pruning we observed might be part of a more generalised vascular injury process in bronchiectasis. Recent studies of subjects with bronchiectasis have documented an increased risk of cardiovascular diseases including myocardial infarction and stroke $[23,24]$. Regardless of the cause of vascular pruning, its existence may partially explain the pulmonary hypertension found in patients with severe bronchiectasis [25]. A large loss of small pulmonary vessels may lead to a shift in blood distribution to central, larger vessels and an increase in the pulmonary artery pressure. Our finding supports further investigation to explore the underlying mechanisms of pulmonary vascular injury in bronchiectasis and its clinical implications.

In an attempt to start to understand the clinical implications of intraparenchymal pulmonary vascular pruning in subjects with bronchiectasis, we examined the association of pruning with FEV1 and 6MWT. We found that those subjects with bronchiectasis and vascular pruning had reduced expiratory airflow and exercise capacity compared to those with bronchiectasis but without vascular pruning. Our findings corroborated a prior observation linking vascular pruning to lower FEV1 and exercise capacity in smokers with and without COPD [4], a disease that shares common clinical features and risk factors with bronchiectasis. Our results highlight the association of vascular loss and those outcomes in bronchiectasis beyond confounding factors such as pack-year of smoking, COPD status and emphysema.

Our study has several limitations. We used a population of heavy smokers who were enrolled into the COPDGene study because their smoking status and the presence of COPD or asthma. Thus, this sample is not representative of the entire smoking or bronchiectasis populations, and caution should be exercised when generalising these findings. However, the rich characterisation of this cohort, including volumetric CT scans allows for exploration of an understudied structure in smokers with radiographic bronchiectasis. Additionally, the particle technique employed to segment and extract the vasculature does not distinguish arteries from veins. In our modelling, we used $\mathrm{BV} 5 / \mathrm{TBV}$ as a metric for vascular pruning; however, the process of vascular loss is likely complex and this metric might not capture this complexity. Additionally, noncontrast CT scans do not allow assessment of the bronchial circulation, so we cannot evaluate the effect of that circulation on our findings. However, since most of our subjects had only mild bronchiectasis, we believe that a bronchial circulation effect did not significantly influence our results. A 
visual assessment to identify bronchiectasis was used and the agreement between readers was only moderate. However, we attempted to reduce diagnosis bias by performing a three-step reading process, involving a second reading for all CTs and a third reading for those CTs in which the primary readers disagreed. We believe that the development of objective methods will likely improve our ability to accurately identify and quantify bronchiectasis.

In summary, we have demonstrated that subjects with bronchiectasis have injury beyond the airways, as demonstrated by intraparenchymal pulmonary vascular pruning. Subjects with bronchiectasis and vascular pruning have impaired expiratory airflows and exercise capacity.

Authors' contributions: All authors conceived and designed the study, and created, revised and gave final approval of this manuscript. A.A. Diaz, G.R. Washko, F. Rahaghi and R. San José Estépar analysed and interpreted the data. A.A. Diaz, F. Rahaghi, D.J. Maselli, A. Yen, Y. Okajima, D.A. Lynch, G.R. Washko and R. San José Estépar acquired the data. A.A. Diaz, D.J. Maselli, F. Rahaghi, C.E. Come, C.H. Martinez, D.A. Lynch and R. San José Estépar drafted the manuscript for important intellectual content. A.A. Diaz and R. San José Estépar take full responsibility for the content of the manuscript, including the data and analysis.

Conflict of interest: A.A. Diaz received research grants from the NIH and Brigham and Women's Hospital, and speaker fees from Novartis Inc. outside of the submitted work. D.J. Maselli has nothing to disclose. F. Rahaghi has nothing to disclose. C.E. Come received NIH/NHLBI grant K23HL114735 during the conduct of the study. A. Yen reports receiving salary support from the NIH under COPDGene R01-HL089897 during the conduct of the study. E.S. Maclean has nothing to disclose. Y. Okajima reports receiving grants from Canon Medical Systems, grants from Ziosoft, outside the submitted work. C.H. Martinez has nothing to disclose. T. Yamashiro has nothing to disclose. D.A. Lynch reports receiving grants from the NHLBI, research supports from Parexel and Veracyte, and personal fees from Boehringer Ingelheim and Genentech/Roche, outside the submitted work. W. Wang has nothing to disclose. G.L. Kinney has nothing to disclose. G.R. Washko reports receiving grants from the NIH, grants and other support from Boehringer Ingelheim, and other support from Genentech, Quantitative Imaging Solutions and PulmonX, during the conduct of the study. G.R. Washko's spouse works for Biogen, which is focused on developing therapies for fibrotic lung disease. R. San José Estépar reports receiving grants from the NHLBI during the conduct of the study. He is founder and co-owner of Quantitative Imaging Solutions, which is a company that provides image-based consulting and develops software to enable data sharing.

Support statement: This work was supported by US National Institutes of Health (NIH) grants (COPDGene; R01HL089897 and R01HL089856); A.A. Diaz is supported by NIH grants K01-HL118714 and R01-HL133137-A1, and the Brigham and Women's Hospital Minority Faculty Career Development Award. The NIH had no role in the design of the study and in the collection, analysis, or interpretation of the data. Funding information for this article has been deposited with the Crossref Funder Registry.

\section{References}

1 Maselli DJ, Amalakuhan B, Keyt H, et al. Suspecting non-cystic fibrosis bronchiectasis: What the busy primary care clinician needs to know. Int J Clin Pract 2017; 71: https://doi.org/10.1111/ijcp.12924.

2 Martinez-Garcia MA, de la Rosa Carrillo D, Soler-Cataluna JJ, et al. Prognostic value of bronchiectasis in patients with moderate-to-severe chronic obstructive pulmonary disease. Am J Respir Crit Care Med 2013; 187: 823-831.

3 McDonnell MJ, Aliberti S, Goeminne PC, et al. Comorbidities and the risk of mortality in patients with bronchiectasis: an international multicentre cohort study. Lancet Respir Med 2016; 4: 969-979.

4 Estepar RS, Kinney GL, Black-Shinn JL, et al. Computed tomographic measures of pulmonary vascular morphology in smokers and their clinical implications. Am J Respir Crit Care Med 2013; 188: 231-239.

5 Santos S, Peinado VI, Ramirez J, et al. Characterization of pulmonary vascular remodelling in smokers and patients with mild COPD. Eur Respir J 2002; 19: 632-638.

6 Ryland D, Reid L. The pulmonary circulation in cystic fibrosis. Thorax 1975; 30: 285-292.

7 Long FR, Williams RS, Castile RG. Structural airway abnormalities in infants and young children with cystic fibrosis. J Pediatr 2004; 144: 154-161.

8 Diaz AA, Young TP, Maselli DJ, et al. Quantitative CT measures of bronchiectasis in smokers. Chest 2017; 151: $1255-1262$.

9 Estepar RS, Ross JC, Krissian K, et al. Computational vascular morphometry for the assessment of pulmonary vascular disease based on scale-space particles. Proc IEEE Int Symp Biomed Imaging 2012: 1479-1482.

10 Regan EA, Hokanson JE, Murphy JR, et al. Genetic epidemiology of COPD (COPDGene) study design. COPD 2010; 7: 32-43.

11 Netter F. Atlas of Human Anatomy. Summit, Ciba-Geigy Corporation, 1989.

12 Stocks J, Quanjer PH. Reference values for residual volume, functional residual capacity and total lung capacity. ATS Workshop on Lung Volume Measurements. Official Statement of The European Respiratory Society. Eur Respir J 1995; 8: 492-506.

13 Gevenois PA, De Vuyst P, de Maertelaer V, et al. Comparison of computed density and microscopic morphometry in pulmonary emphysema. Am J Respir Crit Care Med 1996; 154: 187-192.

14 Hersh CP, Make BJ, Lynch DA, et al. Non-emphysematous chronic obstructive pulmonary disease is associated with diabetes mellitus. BMC Pulm Med 2014; 14: 164.

15 Hankinson JL, Odencrantz JR, Fedan KB. Spirometric reference values from a sample of the general US population. Am J Respir Crit Care Med 1999; 159: 179-187.

16 Wells JM, Estepar RS, McDonald MN, et al. Clinical, physiologic, and radiographic factors contributing to development of hypoxemia in moderate to severe COPD: a cohort study. BMC Pulm Med 2016; 16: 169.

17 Lin LI. A concordance correlation coefficient to evaluate reproducibility. Biometrics 1989; 45: 255-268. 
18 Bland JM, Altman DG. Statistical methods for assessing agreement between two methods of clinical measurement Lancet 1986; 1: 307-310.

19 Ashour M. Hemodynamic alterations in bronchiectasis: a base for a new subclassification of the disease. $J$ Thorac Cardiovasc Surg 1996; 112: 328-334.

20 Martinez-Garcia MA, Perpina-Tordera M, Roman-Sanchez P, et al. Bronquiectasias, inflamación sistémica y factor de necrosis tumoral alfa: factores asociados [The association between bronchiectasis, systemic inflammation, and tumor necrosis factor alpha]. Arch Bronconeumol 2008; 44: 8-14.

21 Wilson CB, Jones PW, O'Leary CJ, et al. Systemic markers of inflammation in stable bronchiectasis. Eur Respir J 1998; 12: 820-824.

22 Saruya S, Yamashiro T, Matsuoka S, et al. Decrease in small pulmonary vessels on chest computed tomography in light smokers without COPD: an early change, but correlated with smoking index. Lung 2017; 195: 179-184.

23 Navaratnam V, Millett ER, Hurst JR, et al. Bronchiectasis and the risk of cardiovascular disease: a population-based study. Thorax 2017; 72: 161-166.

24 Chen YF, Lin HH, Lin CS, et al. Bronchiectasis and increased risk of ischemic stroke: a nationwide population-based cohort study. Int J Chron Obstruct Pulmon Dis 2017; 12: 1375-1383.

25 Wang L, Jiang S, Shi J, et al. Clinical characteristics of pulmonary hypertension in bronchiectasis. Front Med 2016; 10: $336-344$ 